\title{
Editorial
}

\section{Oro-Maxillofacial Radiology and Imaging: An Indispensible Dental Speciality}

\author{
Deepak Gupta ${ }^{*} \$$
}

Department of Oral Medicine and Radiology, M.M. College of Dental Sciences and Research, Mullana, Ambala, Haryana, India

Historically the field of Dentistry was precise and was considered related to dentition only. Now a day, Dentistry is considered as that branch of medicine which is involved in the study, diagnosis, prevention, and treatment of diseases and disorders of not only the Oral cavity, but the maxillofacial region as well. There is no doubt to the fact that Dentistry is widely considered important for the overall health of an individual. This is attributed to the fact that a majority of systemic diseases have a presenting oral sign or a symptom. Furthermore, there is no denying to the fact that Maxillofacial Radiology is considered as the backbone of Dentistry in terms of Investigation, treatment planning as well as follow up. The maxillofacial region is considered as one of the most anatomically complex regions of the body. It extends from the base of the skull to the hyoid bone. However this area contains elements and organs which belong to different systems that can be affected by a variety of local and systemic pathologic processes. Diagnostic Maxillofacial Radiology and Imaging has assumed a lead central role in the evaluation of this region. Fast technologic evolution in the field of Maxillofacial Radiology has made it possible to make utmost accurate and safe diagnosis. This has further led to precise treatment planning as well as follow up. This technological evolution has made Maxillofacial Radiology as an integral part for all the specialities of Dentistry. Although computed tomography in dentistry was a dream 20 years ago, today it has become a very important diagnostic tool. Similarly, the recent introduction of Cone Beam Computed Tomography, Electromagnetic Resonance Imaging Devices and Ultrasonographic with intraoral probes has further upgraded the field of Maxillofacial Radiology.

Owing to the unmatched importance of Maxillofacial Radiology, this particular Guest Edited issue is dedicated to the current research on Oral and Maxillofacial Radiology. Henceforth, I would like to congratulate the vision of the editorial board of The Open Dentistry Journal at Bentham Science Publishers, for realizing a special issue on this topic.

\footnotetext{
*Address correspondence to this author at the Department of Oral Medicine and Radiology, M.M. College of Dental Sciences and Research, Mullana, Ambala, Haryana, India; Pin: 133207; Tel: +91-9896671281;

E-mail: drdeepak_26@rediffmail.com

\$Guest Editor
}

The "Oro-Maxillofacial Radiology and Imaging" is a special thematic issue of "The Open Dentistry Journal" of the Bentham Open and covers the closely related fields of oral radiology and head and neck imaging. This issue has included original peer-reviewed contributions inclined towards the diagnostic oral and maxillofacial radiology and related imaging sciences so as to promote and advance the art and science of radiology in dentistry, and to provide a platform for different Oral Medicine and Maxillofacial radiologists and other experts to highlight their valuable research which may be of use to the healthcare community and to the public. Below is a brief description of the articles of the special issue.

\section{Analysis of Pharyngeal Airway using lateral cephalo- gram vs CBCT images: a cross-sectional retrospective study}

This paper highlights the assessment of correlation between the area and the volume measurements of pharyngeal airway size in a lateral cephalogram and a 3-dimensional (3D) cone-beam computed tomography (CBCT) scan in adolescent subjects. The validity of the information on lateral cephalogram have been questioned because a $2 \mathrm{D}$ representation of a 3-dimensional (3D) structure causes severe limitations as distortion, differences in magnification, superimposition of the bilateral craniofacial structures and lack of information about cross-sectional area and volume. Henceforth CBCT technology can aid in the evaluation of airways and can act as a diagnostic instrument in this area.

2. Evaluation of the root and canal morphology of maxillary permanent molars and the incidence of the second mesiobuccal root canal in Greek population using conebeam computed tomography: A retrospective study.

This article highlights the importance of CBCT in the field of Endodontics. CBCT can be a useful tool to reveal tooth morphology, irregularities, additional root canals and vertical root fractures. The objective of this study was to evaluate the root and root canal morphology of the maxillary permanent molars in Greek population using Cone-Beam Computed Tomography.
3. Correlation Between Clinical Findings Of Temporoman- dibular Disorders And MRI Characterstics Of Disc Dis- placement.


This study evaluated whether MRI findings of various degrees of disk displacement could be correlated with the presence or absence of clinical signs and symptoms of temporomandibular disorders in symptomatic and asymptomatic subjects.

Disk location is of prime importance because the presence of a displaced disk is a critical sign of TMJ dysfunction. However, disk displacement is also frequently seen in asymptomatic volunteers. It is important for the Oral radiologist to detect early MR imaging signs of TMJ dysfunction, thereby avoiding the evolution of this condition to its final stage, which is an advanced and irreversible phase that is characterized by osteoarthritic changes such as condylar flattening or osteophytes.

\section{Effect of Septal Deviation, Concha Bullosa and Haller's Cell on Maxillary Sinus's Inferior Pneumatization; a ret- rospective study}

This study determined the prevalence of nasal septal deviation (NSD), concha bullosa (CB), and Haller's cells (HC) and correlated inferior pneumatization of maxillary sinus (MSIP) with these anatomical variations. Maxillary sinus's inferior pneumatization is a physiological process, which increase over time and accelerates following extraction. It was revealed that NSD, CB and $\mathrm{HC}$ do not have a definite role on sinus's inferior pneumatization.

\section{Relationship between the Thickness of Cortical Bone at Maxillary Mid-palatal Area and Facial Height Using Cone beam Computed Tomography}

Orthodontic mini-implants have been incorporated into orthodontic treatment modalities for anchorage. Since adequate bone at mini-implant placement site can influence the success or failure of anchorage, this present study was done to determine the thickness of cortical bone in the maxillary mid-palatal area at predetermined points for the placement of orthodontic mini-implants using Cone Beam CT technique in order to evaluate the relationship of these values with the facial height.

\section{Evaluation of a Mathematical Model for Digital Image Enhancement}

The purpose of this study was to compare the detected number of holes on a step wedge on images resulting from the application of the 5th degree polynomialmodel compared to the images resulting from the application of linear enhancement.

7. Assessment of the horizontal and vertical position of mental foramen in Indian population in terms of age and sex in dentate subjects by panoramic radiographs: A retrospective study with review of literature ".

This study introduces new criteria to access vertical position of mental foramen in panoramic radiographs in Indian population. Further, the position and symmetry of mental foramen was also determined and in horizontal as well as in vertical plane and the results were compared with those reported for other populations in literature. Further gender differences in mental foramen position were also accessed to comment on the reliability of panoramic radiographs for sex determination.

\section{Evaluation of Radiomorphometric Indices in Panoramic} Radiograph - A Screening Tool

This is a pilot study with an aim to measure the radiomorphometric indices in a digital panoramic radiograph and to find the inter-relationship of the indices with age and sex of the patients. Mandibular cortical indices, like the mandibular cortical index (MCI), panoramic mandibular index (PMI), mental index (MI), antegonial index (AI) and gonial index (GI) have been developed to assess and quantify the quality of mandibular bone mass.

9. Future imaging alternatives: The clinical non-invasive modalities in diagnosis of oral squamous cell carcinoma (OSCC)"

Oral squamous cell carcinoma (OSCC) has a remarkably high incidence worldwide, and a fairly serious prognosis. Henceforth, the dental community especially the Oral radiologists must be well aware of the clinical evaluation of the lesions too along with the imaging findings. Henceforth, In this article, the available Non-imaging methods for diagnosing OSCC have been reviewed. It is clearly evident that screening and early effective detection of cancer and precancerous lesions have the potential to reduce the morbidity and mortality of this disease. The imaging technologies are subjective procedures since all of them require interpretation and are significantly affected by the examiner experience. These make further research for advanced objective procedures are mandatory. Saliva-based oral cancer diagnosis and optical biopsy are promising objective non-invasive methods for diagnosing OSCC. They are easy to perform clinically at primary care set. They show promising pathways for the future development of more effective method for diagnosis of OSCC.

\section{An inventory of current available ultrasound devices for dental use}

This present study is an inventory of the currently available ultrasound imaging devices in the market for dental use. This study also highlights portable ultrasound devices which could be used in general dental offices to image salivary glands, masticatory muscles and lymph nodes. The review specifies that some manufacturers provide portable and nonportable ultrasound devices. The first ones could be valuable in dental settings, as they can easily be moved around in the office. Further ultrasonography is also considered a very good imaging modality for diagnosis of oral lesions like hemangiomas, lymph nodes, metastasis of lesions to lymphnodes etc. This article will be very helpful for the upcoming Oral and Maxillofacial Radiologists as well as general dental practitioners.

\section{Digital DICOM in Dentistry}

Just as in Medicine, digital communication, information processing, and $\mathrm{x}$-ray imaging have changed the face of dentistry. The incorporation of digital systems into medical and dental practice has necessitated development of a standard that allows reliable transmission of information between the devices taking the images, devices storing the images, and devices displaying the images. This standard is termed DICOM. This article briefly reviews how DICOM came about, how dentistry is involved, the various elements that are part of the DICOM system, and how DICOM is currently used in 
dentistry. This article will be very helpful for the general dental practitioners as well as specialists to understand how the modernized radiology has changed and how this digital DICOM in Dentistry can help them in their day to day practice.

\section{Pontine infarct presenting with atypical dental pain: A case report}

This case report highlights a patient who presented with acute onset atypical dental pain with no identifiable dental etiology. A 40 years old male was presented with acute onset, diffuse teeth pain on right side. Dental examination was normal. The patient was further diagnosed as an acute pontine infarct on neuroimaging. Magnetic resonance imaging(MRI) of the brain revealed an acute infarct in right pons near the trigeminal root entry zone. Pontine infarct presenting with dental pain as a manifestation of trigeminal neuropathy, has rarely been reported previously. This stresses the importance of neuroradiology in evaluation of atypical cases of dental pain.

\section{Case Presentation of Concomitant and Contiguous Ade- nomatoid Odontogenic Tumor and Focal Cemento- Ossifying Dysplasia}

This case report highlights diagnosis of two distinct disease processes of different cellular origin namely, focal cemento-ossifying dysplasia and adenomatoid odontogenic tumor in a previously unreported concomitant and contiguous relationship. The diagnosis was determined by a combination of clinical, radiographic, histopathological and surgical evidence. This case highlights two points, first, the need to examine all mixed radiolucent- radiopaque lesions with advanced imaging techniques to assess the number and extent of the lesions prior to treatment planning. Second, a likely role of periodontal ligament as the tissue source for the development of odontogenic tumors and cemento- osseous dysplasias.

The above mentioned articles highlight that Digital imaging, plain and computed tomography, magnetic resonance imaging, ultrasonography and other techniques which are now part of the armamentarium of the dentist, largely through the work of oral and maxillofacial radiologists. Im- provements in the understanding and diagnosis of disease have also been the result of efforts in this specialty. Sometimes diagnosis by conventional radiographs is equivalent to the higher imaging modalities like computed tomography. Henceforth all these imaging modalities must be practised keeping in mind the radiation hazards.

As this thematic issue offers a forum for international collaboration in diagnostic imaging of the dental and maxillofacial region, the coverage has featured cutting-edge research papers, review articles, case reports and technological developments that will surely appeal to clinicians involved in the diagnostic imaging of hard and soft tissue. The contributors to the issue include the researchers and clinicians worldwide.

The editorial board of this special issue of The Open Dentistry Journal features a panel of international expert reviewers who has provided their expertise and guidance in shaping the contents of the issue. Once again I would like to extend my thanks to the Editor in Chief of The Open Dentistry journal for sponsoring this important special issue to uncover the current state-of-the-art scientific literatures and future direction in Maxillofacial Radiology.

\section{ACKNOWLEDGEMENTS}

The contribution of the reviewers was vital for the completion of this special issue. I would like to express my sincere appreciation for the time and constructive comments of the reviewers. Namely, Dr. Amit Mittal MD-Radiodiagnosis, Prof and Head, Dept. of Radiodiagnosis and Imaging, MMU, Mullana; Dr. Nageshwar Iyer MDS, Principal, MMCDSR, Mullana; Dr. Amit Aggarwal, BDS, MDS; Dr. Akanksha Srivastava, Research Associate, Faculty of Dentistry, McGill University, Canada; Dr. Dirk Schulze, DMD, MD, PhD; Dr. Sumanth K, Deputy Dean, Melaka-Manipal Dental College, Melaka; Dr. Daniel Cohen Goldemberg, PhD; Dr. Olga Naka, DDS, PhD; Dr. Mitra Sadrameli, DMD, MS; Dr. Soheyl Sheikh, BDS, MDS; Dr. Shambulingappa P. BDS, MDS; Dr. Durgesh Bailoor, BDS, MDS; Dr. Sunita Gupta, BDS, MDS; Dr. F Karjodkar, MDS; Dr. Jyoti Mago, MDS; Dr. Khallaf Mohd, Dr. Ujjwal Joshi; Dr. Jaisri R Toppay and Dr.Galit Almoznino.

(C) Deepak Gupta; Licensee Bentham Open.

This is an open access article licensed under the terms of the Creative Commons Attribution Non-Commercial License (http://creativecommons.org/licenses/by-nc/3.0/) which permits unrestricted, non-commercial use, distribution and reproduction in any medium, provided the work is properly cited. 